\title{
Editorials
}

\section{RCGP Continuity of Care Toolkit:}

\author{
promoting relational continuity
}

The Royal College of General Practitioners (RCGP) has recently released the Continuity of Care Toolkit' that gives practical advice about how to diagnose and protect the capacity of patients and practitioners to 'retain contact with each other' la nice succinct definition of relational continuity). The toolkit recommends an initial diagnosis of the state of continuity of care in the practice as a trigger for conversations about what specific actions can be taken to safeguard continuity of care. Starting this conversation has just become easier with the availability of a Continuity Calculator, ${ }^{2}$ which helps practitioners generate the needed continuity statistics for the practice.

The toolkit is a welcome response to more than a decade of policy initiatives focusing almost exclusively on improving dimensions of continuity of care that relate to coordinated and consistent management of diseases and facilitated information flow between providers. The need to organise and coordinate care in a way that preserves and promotes relational continuity has not been on the policy radar. From across the Atlantic, we applaud the College's publication of the toolkit and affirm that its relevance extends well beyond the College's UK membership.

\section{RELATIONSHIP-CENTRED CARE}

However, before applying the toolkit to facilitate continuity, it is helpful to remember that continuity is one path towards something more meaningful: relationship-centred care. Relationships are the antidote to an increasingly fragmented and depersonalised healthcare system. ${ }^{3}$ It is easy to forget to value them in a healthcare environment that pays for performance in delivering commodities of care. That same healthcare environment assumes that primary care clinicians take long-term responsibility for patients, but may be subtly devaluing the relationship by focusing on disease care, convenience, and short-term cost reduction over longterm value. It is precisely in this context of a growing need for a relationship, that the RCGP toolkit emerges.

lan McWhinney observed that:

Continuity of care in family practice cannot be adequately described merely in terms of duration ... but involves the family physician's ongoing commitment to the

\section{"The need to organise and coordinate care in a way that preserves and promotes relational continuity has not been on the policy radar.}

\section{patient and his family as persons.'}

\section{VALUING THE RELATIONSHIP}

One empirical study found that the degree to which patients value a continuity relationship with their family physician is indeed predicted by the duration of the relationship. ${ }^{5}$ But as McWhinney intuited, longitudinality of contact is not the only path. This same study found that patients commitment to the relationship also was strongly related to the degree to which they could endorse the statement this physician and I have been through a lot together.' Implied in this is the idea not only of being together for a long time, but being together during key life events: the kind of togetherness that helps to build shared understanding and meaning; that builds trust and a sense of being understood, even when words are not shared. Patients who both have been through a lot with their physician and who have been together for a long time are extraordinarily committed to the relationship. ${ }^{5}$

The RCGP Continuity of Care Toolkit begins by stating that the evidence shows the benefits of continuity for patients and for a high value healthcare system. More subtly stated, but equally important, is that continuity of care matters to the primary care physician. Continuity helps GPs to do their job better and be more confident in managing clinical uncertainty. Although relationships can be a source of consternation (for example, continuity with the heartsink patient), ${ }^{6}$ the ability to be a part of people's lives over time also provides a great source of meaning for the GP and contributes to their professional satisfaction.?

\section{INVESTING IN THE RELATIONSHIP}

The toolkit recognises the challenge of retaining practitioner-patient contact in the context of limited practitioner availability or the imposed constraints of an advanced access system. It alludes to another, often neglected, part of relational continuity: the extension of relationships with trusted colleagues to patients. By defining smaller teams within large practices or having a buddy system with colleagues, physicians can still give patients the security of a relationship even with constrained availability. The toolkit could emphasise more, however, the importance of making this arrangement explicit, thus surrendering the buddy relationship visible to the patient ${ }^{8}$ and to recognising that it can be more challenging to extend the therapeutic relationship to a professional in another discipline, who provides complementary care that is outside the purview of the GP. When patients are out of contact for extended periods, for whatever reason, practitioners can initiate a special visit to reconnect and renew continuity.

Continuity is an investment by both the GP and the patient. Investments in primary care relationships, just like investments in the bank, pay dividends over time. Investment in continuity provides a relationship bank account in which interest accrues from

\section{"The toolkit recognises the challenge of retaining practitioner-patient contact in the context of limited practitioner availability or the imposed constraints of an advanced access system.}




\section{"Continuity is an investment by both the GP and the patient. Investments in primary care relationships, just like investments in the bank, pay dividends over time.}

both large deposits and from many small deposits over time. And when the chips are down - when a new diagnosis, or a family crisis, or infirmity makes patients long to be known as a person - the relationship account yields great rewards during these times of greatest need. It is this relationship asset for which the RCGP Continuity of Care Toolkit provides the investment manual.

Setting up the opportunity for this dividend sometimes requires short-term sacrifices. In health care this often has involved the patient waiting for care or accepting a less than convenient appointment. The RCGP toolkit provides ways to diagnose these tradeoffs from both the patient and the practice point of view, and to prescribe treatment, sometimes using new communication technologies, sometimes organising systems differently.

Continuity provides a foundation for moving to higher levels of care: ${ }^{9}$ for building on the provision of basic care of individual diseases, mental health, prevention, and family care, towards care that is integrated across multiple domains, and personalised and prioritised based on what lona Heath calls balancing the biographical and the biotechnical', and what lan McWhinney calls an acquaintance with the particulars. ${ }^{10}$ Investment in the continuity relationship over time and key events results in a relationship that is healing, but that also involves abiding - sticking it out together even when healing is not forthcoming that is a source of meaning in the end. ${ }^{11}$

\section{Kurt Stange,}

Professor and Gertrude Donnelly Hess Professor of Oncology Research, Departments of Family Medicine, Epidemiology \& Biostatistics, Sociology, and Oncology, Case Western Reserve University, Cleveland, Ohio, US.

\section{Fred Burge,}

Professor and Research Director, Department of Family Medicine, Dalhousie University, Halifax, Nova Scotia, Canada.

\section{Jeannie Haggerty,}

Research Chair in Family and Community Medicine, St Mary's Hospital and Department of Family Medicine, McGill University, Montreal, Quebec, Canada.

\section{Provenance}

Commissioned; not externally peer reviewed.

DOI: 10.3399/bjgp14X679957

\section{ADDRESS FOR CORRESPONDENCE}

Jeannie Haggerty

St Mary's Hospital and Department of Family Medicine, 3777 Jean Brillant. McGill University, Montreal Canada.

E-mail: jeannie.haggertylamcgill.ca

\section{REFERENCES}

1. Royal College of General Practitioners Continuity of Care Toolkit Group. RCGP Continuity of Care Toolkit: Helping clinicians and practices maximise relationship continuity. 2013. http://www.rcgp.org.uk/policy/rcgppolicy-areas/ /media/Files/Policy/A-Z-policy/ Continuity\%20of\%20Care\%20Toolkit.ashx (accessed 6 May 2014).

2. Salisbury C, Murphy M. Continuity of Care Calculator 2013. http://wnw.bristol.ac.uk/ primaryhealthcare/resources/continuityaudit/ laccessed 6 May 2014).

3. Stange KC. The problem of fragmentation and the need for integrative solutions. Ann Fam Med 2009; 7(2): 100-103.

4. McWhinney IR. Continuity of care in family practice. Part 2: implications of continuity. J Fam Pract 1975; 2(5): 373-374.

5. Mainous AG, 3rd, Goodwin MA, Stange KC Patient-physician shared experiences and value patients place on continuity of care. Ann Fam Med 2004; 2(5): 452-454.

6. Smucker DR, Zink T, Susman JL, Crabtree BF. A framework for understanding visits by frequent attenders in family practice. $J$ Fam Pract 2001; 50(10): 847-852.

7. McWhinney IR. Primary care: core values. Core values in a changing world. BMJ 1998; 316(7147): 1807-1809

8. Safran DG. Defining the future of primary care what can we learn from patients? Ann Intern Med 2003; 138: 248-255

9. Heath I, Sweeney K. Medical generalists: connecting the map and the territory. BMJ 2005; 331(7530): 1462-1464.

10. McWhinney IR. 'An acquaintance with particulars .... Fam Med 1989; 21(4): 296-298.

11. Stange KC. A science of connectedness. Ann. Fam Med 2009; 7(5): 387-395. 